High dynamic range temporal contrast measurement and characterization of oscillators for seeding high energy petawatt laser systems

D. Alessi, T. Spinka, S. Betts, V. Kanz, R. Sigurdsson, B. Riordan, J. Crane, C. Haefner

December 2, 2011

CLEO 2012

San Jose, CA, United States

May 6, 2012 through May 11, 2012 
This document was prepared as an account of work sponsored by an agency of the United States government. Neither the United States government nor Lawrence Livermore National Security, LLC, nor any of their employees makes any warranty, expressed or implied, or assumes any legal liability or responsibility for the accuracy, completeness, or usefulness of any information, apparatus, product, or process disclosed, or represents that its use would not infringe privately owned rights. Reference herein to any specific commercial product, process, or service by trade name, trademark, manufacturer, or otherwise does not necessarily constitute or imply its endorsement, recommendation, or favoring by the United States government or Lawrence Livermore National Security, LLC. The views and opinions of authors expressed herein do not necessarily state or reflect those of the United States government or Lawrence Livermore National Security, LLC, and shall not be used for advertising or product endorsement purposes. 


\title{
High Dynamic Range Temporal Contrast Measurement and Characterization of Oscillators for Seeding High Energy Petawatt Laser Systems
}

\author{
D. Alessi, T. Spinka, S. Betts, V. K. Kanz, R. Sigurdsson, B. Riordan, J. K. Crane, and C. Haefner \\ Lawrence Livermore National Laboratory \\ 7000 East Ave., Livermore, CA 94550 \\ Email:alessi2@llnl.gov
}

\begin{abstract}
We have measured the temporal contrast and performance of oscillators to determine their feasibility for future ultra-high-contrast experiments on the Advanced Radiographic Capability at the National Ignition Facility.

OCIS codes: (120.3940) Metrology, (140.790) Ultrafast Lasers
\end{abstract}

Oscillators with high pulse contrast are of interest for energetic laser systems such as the Advanced Radiographic Capability (ARC) [1] at the National Ignition Facility (NIF) [2]. The ARC system will implement chirped pulse amplification (CPA) in a quad of NIF beamlines to produce eight petawatt-class, 3.2-13.6 kJ, short pulse (1-50 ps) laser beams. The ARC laser system is designed for generating high yields of 70-100 keV x-rays by focusing these beams onto targets with intensities of $>10^{18} \mathrm{~W} / \mathrm{cm}^{2}$ and will operate at a center wavelength suitable for seeding the NIF Nd:glass amplifiers $(1053 \mathrm{~nm})$. A high temporal Strehl ratio and contrast are required to achieve these intensities given the unique focusing geometry of $A R C\left(F_{\#}>25\right)$. Pulse cleaning methods such as saturable absorption, XPW and similar techniques that rely on double CPA schemes[3] add complexity and loss to the laser system. It is desirable to start with, and maintain, a high signal-to-noise ratio throughout the system. Therefore, we have evaluated several different commercially available short-pulse oscillators that are based on different mode locking techniques, and directly measured the temporal contrast. Meeting ARC's demanding temporal pre-pulse power requirement $(>80 \mathrm{~dB}$ pre-pulse power contrast at the system output) necessitates a front-end with a power contrast of $>83-85 \mathrm{~dB}$. Future experiments with ARC may require $100 \mathrm{~dB}$ or greater power contrast [4]. To qualify a laser source to this level, we require a measurement dynamic range of $>90 \mathrm{~dB}$. This is challenging due to the limited amount of energy typically available from mode-locked oscillators $(\sim \mathrm{nJ})$. While several temporal contrast measurements of modelocked Ti:sapphire oscillators have been carried out [5-9], there is only a limited amount of published data on oscillators suitable for seeding Nd:glass amplifiers.

To make a direct measurement of the temporal contrast, we employed a SHG scanning autocorrelator with a photomultiplier tube (PMT) detector, paying careful attention to linearity. Optical noise sources such as scatter in the SHG crystal (BBO) and $1 \omega$ light were minimized, and non-optical noise sources were mitigated using a lock-in amplifier with a 13-bit A/D converter and a low noise detector (PMT) with a feedback regulated control voltage. Using these techniques we have achieved a dynamic range of up to $100 \mathrm{~dB}$. Figure 1a depicts the temporal contrast measurement of two different SESAM mode locked, commercial oscillators producing energies of a few nJ. The amplified spontaneous emission (ASE) noise level of each oscillator was measured to be at $77.5 \mathrm{~dB}$ and $87.5 \mathrm{~dB}$. The noise limit was measured by blocking either arm of the autocorrelator during the scan. A scan of \pm 300 ps duration (Figure 1b) shows reflections from both optics in the oscillator and measurement setup. 


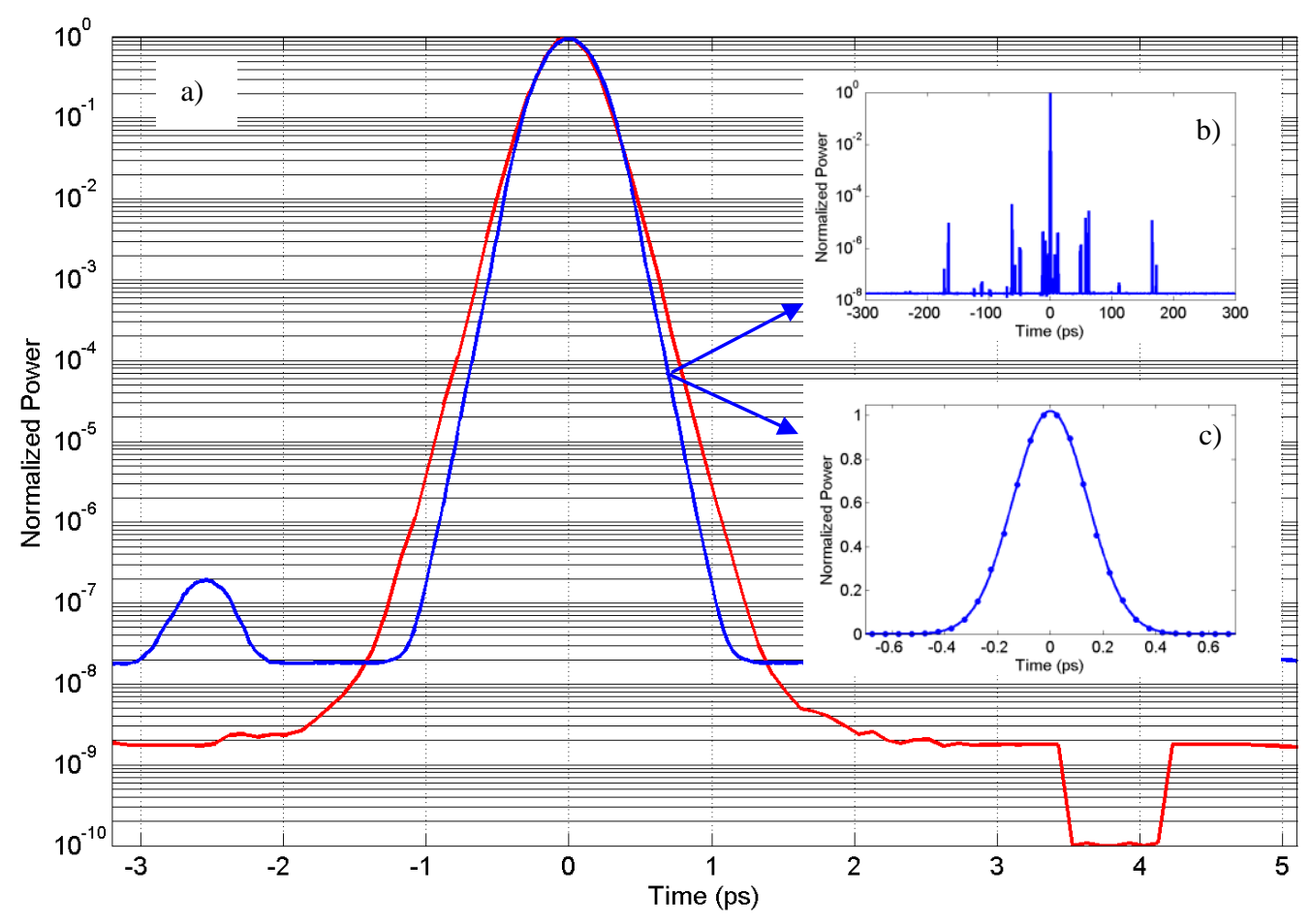

Figure 1: a) High-contrast second order autocorrelation scan of a two SESAM mode locked oscillators. The ASE noise level for the two lasers are measured to be $77.5 \mathrm{~dB}$ and $87.5 \mathrm{~dB}$. The asymmetric dip in the red trace at $4 \mathrm{ps}$ corresponds to a measurement of the noise level of the autocorrelator $(100 \mathrm{~dB})$. The inset graphs show autocorrelation scans with a range of \pm $300 \mathrm{ps}$ (b) and a linear scale with a fit (c) for one of the oscillators.

We have carried out precision characterization of a variety of sources for seeding short-pulse high energy petawatt laser systems like ARC. We will report on the temporal characteristics and performance of several oscillators, both commercially available and those developed at LLNL, evaluating their suitability as sources for future ultra-highcontrast laser systems.

This work was performed under the auspices of the U.S. Department of Energy (DOE) by Lawrence Livermore National Laboratory under contract DE-AC52-07NA27344.

\section{References:}

[1] C.P.J. Barty, et al., "An overview of LLNL high-energy short-pulse technology for advanced radiography of laser fusion experiments,” Nucl. Fusion 44, S266-S275 (2006)

[2] C.A. Haynam, et al., "National Ignition Facility laser performance status," Appl. Opt. 46, 3276-3303 (2007)

[3] M. P. Kalashnikov, E. Risse, H. Schönnagel, and W. Sandner, "Double chirped-pulse-amplification laser: a way to clean pulses temporally," Opt. Lett. 30, 923-925 (2005)

[4] D. Homoelle, J. K. Crane, M. Shverdin, C. L. Haefner, and C. W. Siders, "Phasing beams with different dispersions and application to the petawatt-class beamline at the National Ignition Facility," Appl. Opt. 50, 554-561 (2011)

[5] P.F. Curley, G. Darpentigny, G. Cheriaux, J.P. Chambaret, A. Antonetti, "High dynamic range autocorrelation studies of a femtosecond Ti:sapphire oscillator and its relevance to the optimisation of chirped pulse amplification systems," Opt. Comm. 120, 71-77 (1995)

[6] I.D. Jung, F.X. Kärtner, J. Henkmann, G. Zhang, U. Keller, "High-dynamic-range characterization of ultrashort pulses," Appl. Phys. B. 65, 307-310 (1997)

[7] M. Nantel, et al., "Temporal contrast in Ti:sapphire lasers: characterization and control," IEEE J. Select Topics Quantum Electron. 4, 449-458 (1998)

[8] A. Kasper and K.J. Witte, "Contrast and phase of ultrashort laser pulses from Ti:sapphire ring and Fabry-Perot resonators based on chirped mirrors,” J. Opt. Soc. Am. B 15, 2490-2495 (1998)

[9] A. Braun, et al., "Characterization of short-pulse oscillators by means of a high-dynamic-range autocorrelation measurement,” Opt. Lett. 20, 1889-1891 (1995) 